\title{
An examination of the automated design of prestressed concrete bridge decks by computer
}

\author{
D. BOND
}

\section{Dr J. B. Bellamy, South Yorkshire County Council}

The Paper illustrates the dichotomy that can exist in the engineering profession between the research specialist and the practising designer. To a bridge designer there are some unacceptable points of philosophy and detail which would prevent the use of the computer program in a real situation.

44. The Author has idealized the bridge as a continuous beam, i.e. he is only considering longitudinal effects and in particular is neglecting the problems of bridge design such as transverse distribution and shear lag (\$5). Practical experience shows that for most bridges it is the HB vehicle, not the HA loading, which governs the overall design. However, the Author appears to regard the HB loading as causing local effects only $(\S 33)$. At present bridges are designed to satisfy Department of the Environment memoranda not British Standard codes of practice ( $\$ \S 3$ and 9$)$.

45. The whole Paper is based on construction rates obtained from one bridge project by one contractor. Under normal tendering procedures it is normal for six firms to tender for a bridge construction contract; a detailed examination of the submitted tenders is extremely illuminating in that frequently the only similarities between different tenderers is the final contract price, it being normal for individual rates for any bill item to vary by large percentages. This variation arises because of different methods of allocating construction costs and overheads to unit rates, proposed methods of working and desired cash flows during the contract. Is the program sensitive to markedly different ratios of unit costs?

46. With regard to the examples in the Paper, it appears that the program has a propensity to select narrow webs and curved soffits; although these are economical in material they introduce many problems for the contractor who would certainly tender higher unit rates than for a constant depth section with relatively thick webs. Does the Author intend to extend this program so that the rate selected is commensurate with the difficulty of the task?

47. The program also removes control of the appearance of the bridge from the designer to a minimum cost function stored in a machine; the Author appears to hope that the result will be pleasing (\$2). Bridge design involves the balancing of many nonquantifiable aesthetic factors and as such is an art not a branch of numerical science. I would suggest that the Author should consider introducing the external shape of the bridge into his program and using this as a constraint to his iterations; the program would then be considering the optimum thickness of webs, fianges and diaphragms and this would be of great interest to the bridge designer.

Paper published: Proc. Instn Cio. Engrs, Part 2, 1975, 59, Dec., 669-697. 


\section{Professor Bond}

Dr Bellamy's comments will be particularly useful when this program is being extended.

49. The longitudinal effects of $\mathrm{HB}$ loading could be included without much difficulty, but when writing the initial program I was influenced by the statement in Appendix $F$ of reference 3, which recommends that $H A$ loading can represent full $\mathrm{HB}$ loading on prestressed concrete deck slabs provided that théy have spans greater than $12 \cdot 2 \mathrm{~m}$. While recognizing that bridges are designed to satisfy Department of the Environment memoranda, it was thought that amendments to these publications could be influenced by the new codes of practice ${ }^{1-3}$ and that it would be advisable to anticipate these in the program.

50. Dr Bellamy discusses the unit rates of bill items in tenders in $\$ 45$ and raises a complex problem which I tried to avoid by using arbitrary but consistent unit rates for comparing different designs. It is clear that the designer's organization should estimate the cost of his final design by attempting to predict the value of the successful tender and its corresponding final cost. To what extent unit rates of bill items should influence the character of the design is more difficult to decide. Does the use of measured rates without overheads in a design search give the best allocation of resources of a highway authority, or are these rates influenced excessively by the possibly temporary skills or plant which the contractor has available for that project? Should the designer be influenced by the expected capabilities in the short term of particular contractors or should he develop design styles which he considers will be most economical to construct on different occasions in the long term? It might also be asked whether such close attention to economy is justified in all cases.

51. The program has not yet been applied to a systematic examination of unit costs. One example of altering costs of tenders and formwork by $25 \%$ was presented in $\$ 21$, but in view of Dr Bellamy's remarks this percentage might be inadequate.

52. It is possible to include empirical expressions for unit rates in the program so that they are commensurate with the difficulty of the task $(\$ 46)$ but this would not be satisfactory if any of these were discontinuous, causing steps in the cost functions. For example, there would be no continuous transition from unit costs for a deck with curved shuttering to a design having a constant depth section and straight formwork. It would be necessary to design curved and straight decks separately and to compare their final costs.

53. I admit that the appearance of most of the examples in the Paper was controlled by the program ( $\S 47)$, by way of demonstration. However, during a design search any structural dimension can retain its value which is chosen by the designer.

54. A complete program which designs automatically any prestressed or reinforced concrete bridge deck in its entirety would be required to include a complete selection of methods of structural analysis and all the rules of relevant codes of practice which have been translated into its programming language. In addition, it would need to incorporate the knowledge of the best bridge designers in complete detail with rules for its application in all circumstances.

55. It is clearly impossible to write such an idealized design program at present, and as a consequence I wrote the simple program described in the Paper, which will hopefully enable some characteristics of bridge designs to be compared although only longitudinal effects are considered. If the program is to be used to design webs, flanges and diaphragms in detail, as suggested by Dr Bellamy, a more comprehensive structural analysis will be required, and this could be the next stage in the development of the program. 